\title{
Synthesis of a monophasic nanohybrid for a controlled release formulation of two active agents simultaneously.
}

\begin{abstract}
A new monophasic nanohybrid containing two phenoxyherbicides anions, 4-(2,4dichlorophenoxy)butyrate (DPBA) and 2-(3-chlorophenoxy)propionate (CPPA), was successfully synthesized. The two anions were simultaneously intercalated into the inorganic interlayers of zinc-aluminum-layered double hydroxide (ZAL) for the formation of a nanohybrid, ZAL-CPPA-DPBA (NCDX) by an anion-exchange method. Powder X-ray diffraction, direct-injection mass spectrometry and Fourier transform infrared spectroscopy studies confirmed the dual intercalation of both anions into the host interlayer regions. The well-ordered, layered nanohybrid was synthesized using 0.1 . M solution of both anions. The basal spacing expanded from 8.9. $\AA$ in ZAL to 27.43. $\AA$ in the nanohybrid. The release of both anions from the nanohybrid into an aqueous solution of 0.001 . M sodium carbonate was found to be governed by pseudo second-order kinetics. This release demonstrates the potential application of layered materials as reservoirs and delivery system, especially when multiple active agents are used simultaneously in the formation of an organic-inorganic hostguest nanohybrid with controlled-release properties.
\end{abstract}

Keyword: Monophasic; Nanohybrid; Controlled release formulation; Release of two active agents simultaneously. 